Indonesian Journal of Physics

Vol 20 No. 3, October 2009

\title{
Imaging Subsurface Fracture and Void using Wide-Band Inversion of Fresnel Interpolated Wavepath Seismic Tomography
}

\author{
Bagus Endar B. Nurhandoko \\ Wave Inversion and Subsurface Fluid Imaging Research Lab. (WISFIR Lab) \\ Department of Physics, Institut Teknologi Bandung \\ e-mail:bagusnur@bdg.centrin.net.id
}

\begin{abstract}
Studying existence of fracture, void as well as fault in subsurface are important and it has wide applications. In addition, fracture and void play important role in reservoir productivity as well as in geotechnical application. The problem in imaging fracture and void in subsurface, however, is resolution. It needs high-resolution imaging method laterally as well as vertically.

The seismic velocity imaging, however, usually has poor lateral resolution. Seismic tomography provides better resolution than conventional velocity analysis methods. Conventional raypath seismic tomography, however, needs dense as well as high angle and wide measurement configuration that is high cost in field data acquisition.

I propose to use Fresnel interpolated wavepath (FIW) wide-band inversion. FIW is an interpolation between imaginary part of Rhytov scattering wavepath and raypath. Then, FIW is combined with wide band inversion procedure to handle sparseness configuration of measurement. By this method, smooth constraint is implemented more naturally by based on wave's spectrum. Furthermore, it can produce more stable inversion procedure. In this paper, Wide-band inversion of FIW tomography is tested into various seismic field data in order to image void, fault and fracture. The results showed that tomography based on FIW could handle the limitation of configuration angle, sparseness configuration and resulting good image. We use many difficult cases of field data such as: subsurface tunnel imaging and also void-fracture imaging in volcanic rock
\end{abstract}

Keyword: Seismic tomography, Fresnel interpolated wavepath, Rhytov scattering, fracture and void imaging, Wideband inversion

\section{Introduction}

Seismic tomography is developed from tomography technique that initiated in early 1960's where Honsfeld and Cormack received Noble prize in medicine in 1979. The seismic tomography is used for imaging seismic velocity of subsurface. Several seismic tomography techniques have been already used recently. One of them is travel time seismic tomography that uses travel times information to calculate velocity distribution of subsurface. Seismic tomography uses difference of travel times of the observation data and those of the model. The travel times of the model are calculated from the integral of slowness and path-length over the raypath, while travel times of the observation data are picked as the first breaks of the transmitted waves. Some of tomography methods have been proposed using raypath information ${ }^{1-3)}$.

Ray-trace tomography works well when the following two requirements are satisfied ${ }^{4)}$. First, because the ray relies on the high frequency assumption of ray theory, the velocity data to be examined must vary slowly compared with the scale of the source wave length. In this case, there is no scattering, phase delay is linear with frequency, the source wavelet is not distorted, and seismic events are completely characterized by travel times. Secondly, without scattering, rays is a very narrow in space, and the source-geophone geometry must provide many view angles through the medium. When these requirements are not satisfied, wave theoretic tomography provides a better image.

Wave theoretic tomography called diffraction tomography is usually formulated in the frequencywavenumber domain ${ }^{4,5)}$. Woodward ${ }^{6)}$ reformulated the wave theoretic tomography to makes wave equation tomography more flexible than diffraction tomography in dealing with irregularly sampled survey data.

In this paper, I propose a new wavepath that combining scattering wavepath and raypath (nonscattering wavepath). Some test in field data showed good results in inversion robustness.

\section{Scattering Wavepath and Fresnel Zone Interpolation Wavepath}

In the seismic wave propagation, a wave propagates like a wavepath that is more natural than a raypath. In the high frequencies the shape of the wavepath becomes thin like a raypath. In the spacetime domain, two techniques have been proposed by Vasco et. al., ${ }^{7)}$. The first is wavepath tomography calculation based on Born approximation by computing the Frechet derivatives (perturbation of waveform with respect to elastic parameter). The second is wavepath approximated by the function of area of the Fresnel ellipse which is perpendicular to the ray, the area of the Fresnel is determined by 
paraxial ray approximation, both wavepaths is quite similar.

In this paper, we present the inversion algorithm of travel time tomography which is formulated from interpolation of scattering wavepath and raypath, we then named this wavepath as Fresnel zone interpolation wavepath. The computation is done in time-space domain.

In the seismic wave propagation, a wave propagates like a wavepath which is more natural than a raypath. However, the shape of the wavepath also becomes thin like a raypath at high frequencies. The wavepath is approximated by Fresnel zone wavepath derived from the linear relationship between slowness and wave field phase perturbation. It can be derived from the first order of Rhytov approximation ${ }^{5)}$.

$$
\left.\left.\Delta \phi(g \mid s)=\int_{A}\left(\frac{G_{b}(g \mid r) \psi_{0}(s \mid r)}{\psi_{0}(s \mid g)}\right)(\nabla(\Delta \phi(s \mid r, Q r)))^{2}+Q r\right)\right) d r
$$

Where $\Delta \phi$ indicates the wave field phase perturbation. $\mathrm{O}(\mathrm{r})$ denotes perturbed slowness field which is approximated by equation (2).

$$
O(r) \approx 2 k_{o}^{2} \frac{\Delta p}{p_{o}}
$$

Where $\mathrm{p}_{\mathrm{o}}$ is slowness, $\mathrm{k}_{0}$ is wavenumber and $\mathrm{G}_{0}(\mathrm{r} \mid \mathrm{g})$ is the Green's function of wave propagating from receiver $(\mathrm{g})$ to any point $(\mathrm{r})$. We express $\mathrm{G}_{0}(\mathrm{r} \mid \mathrm{g})$ as a function of travel time and phase difference.

$$
G_{0}(g \mid r)=\omega_{0} p_{0} \frac{e^{i \omega t(g \mid r)}}{\Delta \Phi}
$$

Where, $\omega_{0}$ is angular frequency and $\Delta \Phi$ is the phase difference between receiver's phase and $\mathrm{r}$ point's phase. $\Psi_{0(s \mid r)}$ and $\Psi_{0(s \mid g)}$ indicate normalized wave field function propagating from receiver to any point (r) and from source to receiver respectively, expressed by equation (4).

$$
\begin{aligned}
& \psi_{0}(s \mid g)=e^{i \omega_{0} t(s \mid g)}, \\
& \psi_{0}(s \mid r)=e^{i \omega_{0} t(s \mid r)}
\end{aligned}
$$

Equation (1) is quite difficult to interpret physically. However, under the Rhytov approximation $[\nabla(\Delta \phi)]^{2}<<O(r)$, it can be simplified ${ }^{5,6)}$.

$$
\Delta \phi(g \mid s)=\int_{A}\left(\frac{G_{0}(g \mid r) \psi_{0}(r \mid s)}{\psi_{0}(g \mid s)}\right){ }_{\mathrm{Im}} O(r) d r
$$

$\Delta \phi(\mathrm{g} \mid \mathrm{s})$ of equation (5) is the complex phase difference between the normalized observation wave field $\psi_{\text {observation }}$ and the normalized calculated wave field $\psi_{\text {calculated, and that is defined as }}$ $\ln \left(\psi_{\text {observation }} / \psi_{\text {calculated }}\right) . \quad \mathrm{G}_{0}$ and $\Psi_{0}$ are complex functions also (equation (3) and equation (4)). To fulfill the definitions, we consider only the imaginary part of the function in the parenthesis of equation (5) which is indicated by subscript Im. By substituting the slowness perturbation $\mathrm{O}(\mathrm{r})$ of equation (2) into equation (5), and substituting wavenumber parameter $\left(\mathrm{k}_{0}\right)$ by angular frequency $\left(\omega_{0}\right)$ and slowness $\left(\mathrm{p}_{0}\right)$, the equation becomes equation (6).

$$
\Delta \phi(g \mid s)=\int_{A} 2 \omega 0^{2} p_{0} G(r \mid g, s) \Delta p \cdot d r
$$

Where $\mathrm{G}(\mathrm{r} \mid \mathrm{g}, \mathrm{s})$ is expressed by equation (7).

$$
G(r \mid g, s)=\left(\frac{G_{0}(g \mid r) \psi_{0}(r \mid s)}{\psi_{0}(g \mid s)}\right)_{\operatorname{Im}}
$$

The receiver and source being interchanged by the reciprocity principle. Therefore in order to make the equation (7) satisfy the boundary condition, the spreading parameter $\Delta \Phi$ in $\mathrm{G}_{0}(\mathrm{~g} \mid \mathrm{r})$ of equation (3) is approximated by $\omega_{0}\left(\mathrm{~T}_{\mathrm{sr}}+\mathrm{T}_{\mathrm{gr}}-\mathrm{T}_{\mathrm{sg}}\right)$, where $\mathrm{T}_{\mathrm{sr}}$ is travel time from source to point $r, T_{g r}$ is travel time from receiver to point $r$ and $T_{s g}$ is travel time from source to receiver. By this approximation resulted in the $\mathrm{G}(\mathrm{r} \mid \mathrm{g}, \mathrm{s})$ function of equation (7) becoming maximum at the shortest path's position (when $\mathrm{T}_{\mathrm{sr}}+\mathrm{T}_{\mathrm{gr}}$ is equal with $\mathrm{T}_{\mathrm{sg}}$ ). The travel times are calculated by means of the reciprocity shot technique ${ }^{8)}$.

Here, we consider only the imaginary part of $\mathrm{G}(\mathrm{r} \mid \mathrm{g}, \mathrm{s})$ in equation (7), by substituting the equations (3) and (4) into equation (7), then I propose this wavepath to be interpolated with raypath, so $G(r \mid g, s)$ can be expressed simply as sinc function by equation (8).

$$
G(r \mid g, s)=\omega_{0} p_{0} \sin c\left(\omega_{0} . \Delta \tau\right)
$$

Where, $\Delta \tau$ indicates travel time from source to any point (r) added to travel time from any point (r) to receiver and subtracted by travel time from source to receiver $\left(\Delta \tau=\mathrm{T}_{\mathrm{sr}}+\mathrm{T}_{\mathrm{rg}}-\mathrm{T}_{\mathrm{sg}}\right)$.

The inversion formula based on Fresnel zone wavepath for estimating the slowness field is derived from equation (6) into the discreet model as written by equation (9).

$$
\Delta \phi(g \mid s)=\sum_{r}\left(2 . \omega_{0}^{2} p_{0} G(r \mid s, g)\right) \Delta p . d
$$

where $d$ is a raypath length in a cell, and can be approximated as grid length of cell. In order to minimize the slowness difference $\Delta \mathrm{p}$, phase misfit $\Delta \phi$ is minimized by equation (9), so the method of Lagrange multiplier is useful to minimize these. By using equation (9), the error function $(E)$ of Lagrange multiplier method is expressed as follows:

$$
E=\sum\left((\Delta p)^{2}-L_{\lambda} A(k)\left(2 \omega_{0}{ }^{2} p_{0} G(r \mid g, s) \Delta p . d-\Delta \phi\right)\right)
$$

These errors are summed to the whole area. $L_{\lambda}$ is a constant of Lagrange multiplier and $\mathrm{A}(\mathrm{k})$ is the arbitrary weight factor. To minimize the error function against slowness perturbation $(\Delta p)$, the error function $\mathrm{E}$ is minimized by differentiating error function against $\Delta \mathrm{p}$. 
Let's assume wave monochromatic, that is $\Delta \phi$ $=\omega_{0} \Delta T$, where $L_{\lambda}$ is angular frequency and $\Delta T$ is travel time difference $\left(\Delta T=\mathrm{T}_{\text {observation }}-\mathrm{T}_{\text {calculated }}\right)$ between observed travel time and calculated travel time for the model. Therefore, at the minimum of error function $\mathrm{E}$ (equation (10)), the slowness perturbation $(\Delta \mathrm{p})$ is expressed by equation (11),

$$
\Delta p=\frac{\omega_{0} 4 \cdot p_{0}{ }^{2} \Delta T \cdot A(k) \cdot d \cdot \sin c\left(\omega_{0} \cdot \Delta \tau\right)}{\sum_{r}\left(2 \cdot \omega_{0} 6 \cdot p_{0} 4 \cdot \sin c^{2}\left(\omega_{0} \cdot \Delta \tau\right) \cdot d^{2} \cdot A(k)^{2}\right)}
$$

When the arbitrary weight factor is assumed as $A(k)=\left(2 \omega_{0}{ }^{2} p_{0}{ }^{2}\right)^{-1}$, the inversion formula can be expressed as follows:

$$
\Delta p=\frac{d \cdot L_{\text {wavepath }} \cdot \Delta T}{\sum_{r} d^{2} \cdot L_{\text {wavepath }}^{2}}
$$

Where the Lwavepath is Fresnel interpolated wavepath that interpolate between scattering Rhytov wavepath and raypath expressed by equation (13)

$$
L_{\text {wavepath }}=\sin c\left(\omega_{0} . \Delta \tau\right)
$$

\section{Application in Subsurface Tunnel Imaging using Zero Degree Aperture angle}

The main problem in tomography is aperture angle and horizontal seismic has zero degree aperture angle. This configuration tends resulting local minimum inversion. Here, an imaging of subsurface tunnel using Fresnel zone interpolation wavepath to the real field data was done to test the performance of this algorithm.

The tunnel location is near Sabuga ITB, Bandung, which is shown by Figure 1. We deployed land seismic cable including geophones on the land surface using 24 geophones above the tunnel (Figure 2). Sources were generated at each position of geophones and some shots were generated on the far position.

The inversion of travel times is done using wide-band FIW $5 \mathrm{~Hz}$ to $60 \mathrm{~Hz}$, and the inversion result is shown in Figure 3. Some events in the subsurface can be detected clearly such as: position of tunnel and pillar positions.

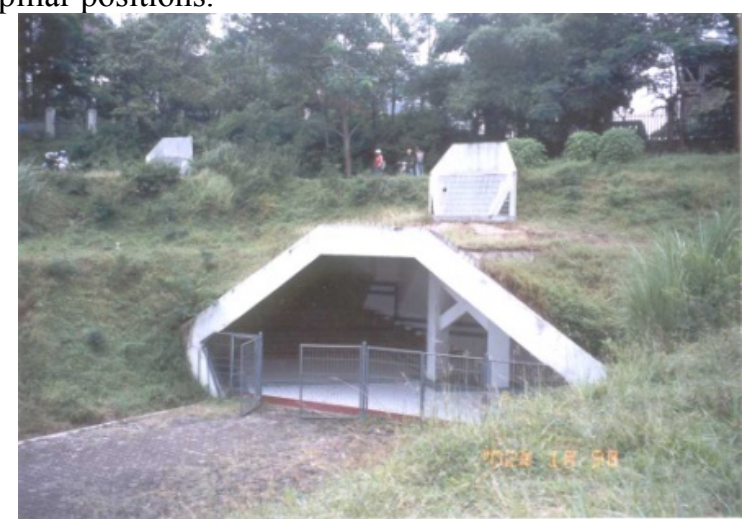

Figure 1. ITB Sabuga Tunnel

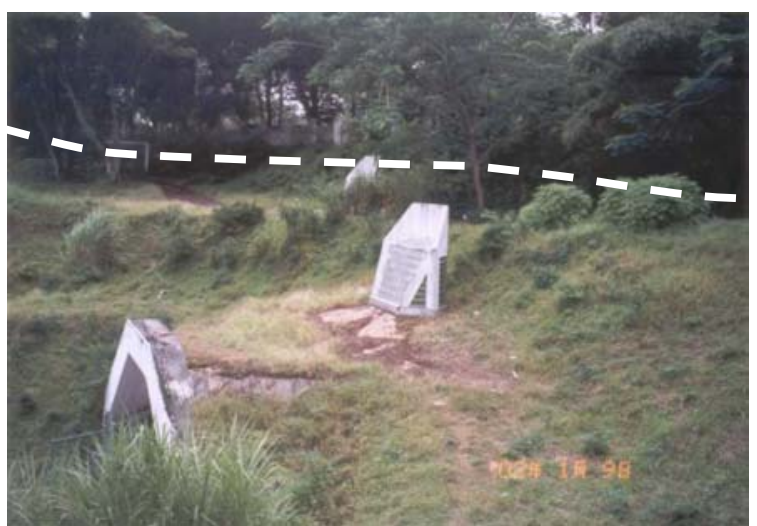

Figure 2. Seismic line for tomography acquisition (white dashed line showed the position of geophones).

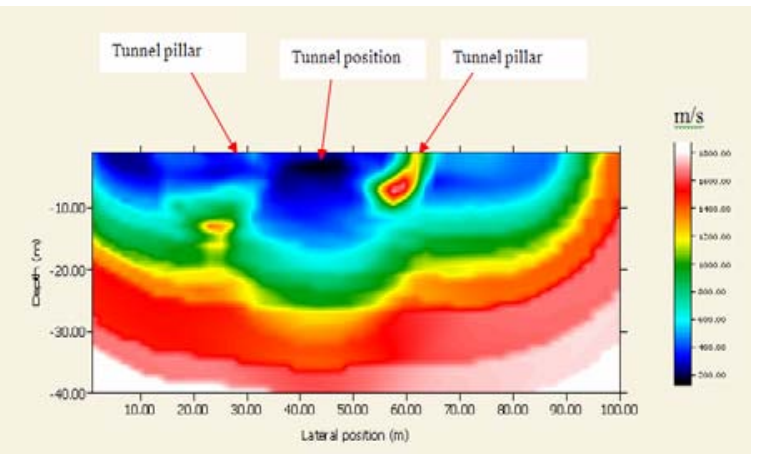

Figure 3. Subsurface velocity tomogram of tunnel

\section{Fracture Identification in Volcanic Rock Sstructure}

This seismic tomography algorithm was applied to the real field data in order to identify natural fracture. Data were collected using Seistronix RAS 24 ( 24 bits) that is completed by 24 geophones and take out cables.

Figure 4 shows drilling rock to put geophones. After lying geophones, seismic wave is generated by hitting rock using hammer or fire crackers (Figure 5).

Geophones are laid surround rock structure, so that we can reach maximum aperture angle and dense configuration. There are shots in every position of receivers and some shots are done on the far place surrounding the volcanic rock hill.

Then, data are inverted by Fresnel interpolated wavepath tomography in wide spectrum frequencies from $5 \mathrm{~Hz}$ to $100 \mathrm{~Hz}$. We can see in figure 6 . the inversion result that void and fractures is clearly identified. 


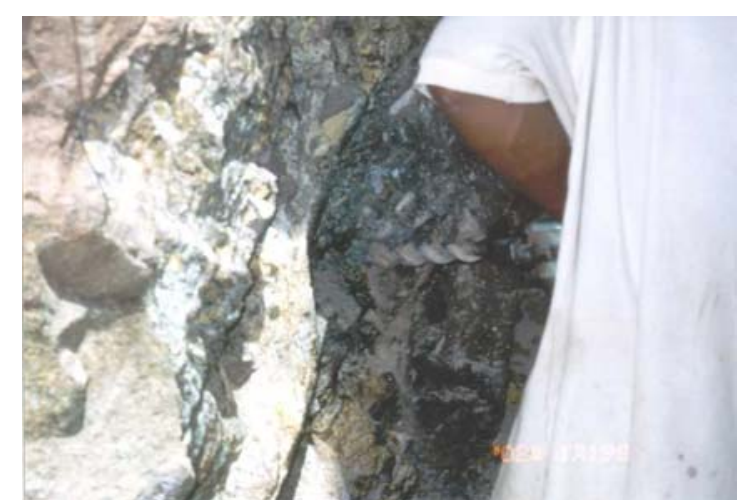

Figure 4. Drilling rock structure to put geophone on the rock

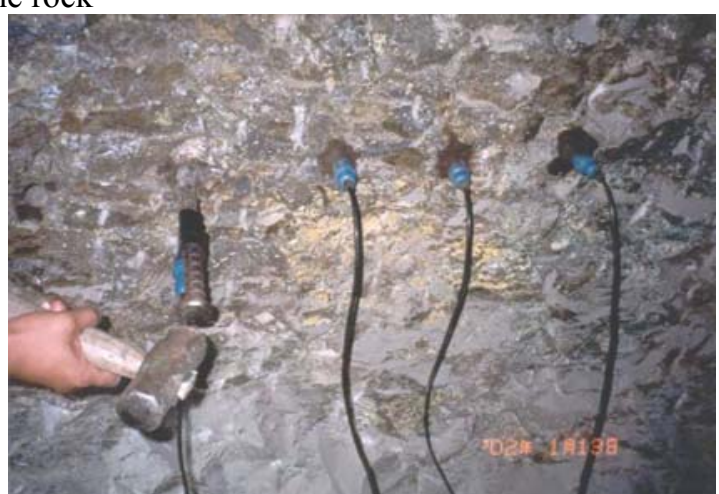

Figure 5. Seismic wave is generated by knocking the rock in every positions of rock and bursting the fire crackers in far positions

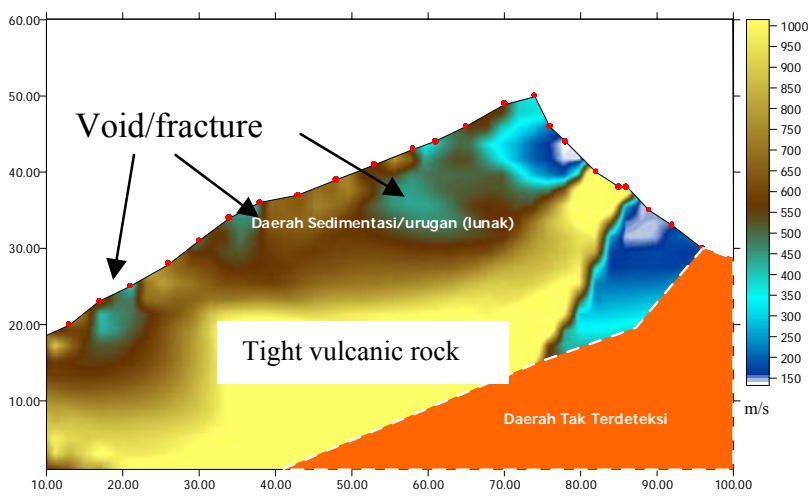

Figure 6. Velocity image of volcanic rock structure

\section{Conclusion}

I show that seismic tomography using wideband Fresnel interpolated wavepath (FIW) can handle difficult case of tomography imaging,i.e: imaging void and fracture in zero aperture tomography. We can easily identify void in rock structure because the void which filled by air usually has low velocity (around $360 \mathrm{~m} / \mathrm{s}$ ) and the other hand the tight rock usually has high velocity $(2300 \mathrm{~m} / \mathrm{s}-4000 \mathrm{~m} / \mathrm{s})$. Zero aperture tomography also promises reduction in acquisition cost for reconstructing void in subsurface. This FIW tomography algorithm works well for imaging the subsurface in difficult cases, i.e: subsurface imaging od void using zero aperture tomography and fracture imaging in volcanic rock.

\section{References}

1. R.D. Radcliff and C.A. Balanis, Reconstruction Algorithms for Geophysical Application in Noisy Environment, Proc. IEEE, 67, 1060-1065, 1979.

2. G.T. Herman, Image Reconstruction from Projections: the Fundamental of Computed Tomography, Academic Press., 1980

3. C.L. Morgan, Basic Principles of Computed Tomography, University Park Press., 1983.

4. R. $\mathrm{Wu}$ and M.N. Toksoz, Diffraction Tomography and Multisource Holography Applied to Seismic Imaging, Geophysics, 52, 1125, 1987.

5. M. Slaney, A.C. Kak and L.E. Larsen, Limitations of Imaging With First-Order Diffraction Tomography, IEEE Trans. on Microwave Th. and Tech., 32, 860-874, 1984.

6. M.J. Woodward, Wave-equation Tomography, Geophysics, 57,15-26, 1992.

7. D.W. Vasco, J.E. Peterson, Jr. and E.L. Majer, Beyond Ray Tomography: Wavepaths and Fresnel Volumes, Geophysics, 60, 1790-1804, 1995.

8. T. Matsuoka and T. Ezaka, Ray Tracing Using Reciprocity, Geophysics, 57, 326-333, 1992. 